\title{
Extreme ultraviolet ionization of pure He nanodroplets: Mass-correlated photoelectron imaging, Penning ionization, and electron energy-loss spectra
}

\author{
D. Buchta, ${ }^{1}$ S. R. Krishnan, ${ }^{2, a)}$ N. B. Brauer, ${ }^{3}$ M. Drabbels, ${ }^{3}$ P. O'Keeffe, ${ }^{4}$ M. Devetta, ${ }^{5}$ \\ M. Di Fraia, ${ }^{6}$ C. Callegari, ${ }^{7}$ R. Richter,${ }^{7}$ M. Coreno, ${ }^{4}$ K. C. Prince,${ }^{7}$ F. Stienkemeier ${ }^{1}$ \\ J. Ullrich, ${ }^{2,8}$ R. Moshammer, ${ }^{2}$ and M. Mudrich ${ }^{1, b)}$ \\ ${ }^{1}$ Physikalisches Institut, Universität Freiburg, 79104 Freiburg, Germany \\ ${ }^{2}$ Max-Planck-Institut für Kernphysik, 69117 Heidelberg, Germany \\ ${ }^{3}$ Laboratoire de Chimie Physique Moléculaire, Swiss Federal Institute of Technology Lausanne (EPFL), \\ 1015 Lausanne, Switzerland \\ ${ }^{4}$ CNR Istituto di Metodologie Inorganiche e dei Plasmi, CP10, 00016 Monterotondo Scalo, Italy \\ ${ }^{5}$ CIMAINA and Dipartimento di Fisica, Università di Milano, 20133 Milano, Italy \\ ${ }^{6}$ Department of Physics, University of Trieste, 34128 Trieste, Italy \\ ${ }^{7}$ Elettra-Sincrotrone Trieste, 34149 Basovizza, Trieste, Italy \\ ${ }^{8}$ Physikalisch-Technische Bundesanstalt (PTB), Bundesallee 100, D-38116 Braunschweig, Germany
}

(Received 15 May 2013; accepted 30 July 2013; published online 22 August 2013)

\begin{abstract}
The ionization dynamics of pure He nanodroplets irradiated by Extreme ultraviolet radiation is studied using Velocity-Map Imaging PhotoElectron-PhotoIon COincidence spectroscopy. We present photoelectron energy spectra and angular distributions measured in coincidence with the most abundant ions $\mathrm{He}^{+}, \mathrm{He}_{2}^{+}$, and $\mathrm{He}_{3}^{+}$. Surprisingly, below the autoionization threshold of $\mathrm{He}$ droplets, we find indications for multiple excitation and subsequent ionization of the droplets by a Penning-like process. At high photon energies we observe inelastic collisions of photoelectrons with the surrounding He atoms in the droplets. (c) 2013 AIP Publishing LLC. [http://dx.doi.org/10.1063/1.4818531]
\end{abstract}

\section{INTRODUCTION}

Helium nanodroplets are intriguing many-body quantum systems which feature special properties such as very low equilibrium temperature $(0.38 \mathrm{~K})$, superfluidity, and the ability to efficiently cool and assemble embedded species (“dopants"). Therefore, pure $\mathrm{He}$ nanodroplets have been extensively studied using electron impact ionization ${ }^{1-5}$ as well as by photoexcitation and ionization with synchrotron radiation. ${ }^{6-11}$ Recently, time-resolved experiments have become possible using femtosecond light pulses in the extreme ultraviolet (EUV) spectral range provided by high-order harmonic generation. ${ }^{12-15}$ Based on the photoionization and dispersed fluorescence emission measurements, the following three distinct regimes of excitation and ionization have been identified:

(i) At photon energies $20.5<h v<23 \mathrm{eV}$, He nanodroplets are excited with large cross sections into perturbed excited states ("bands") derived from the $1 \mathrm{~s} 2 \mathrm{~s}^{1} \mathrm{~S}$ and $1 \mathrm{~s} 2 \mathrm{p}^{1} \mathrm{P} \mathrm{He}$ atomic levels. Fast droplet-induced intraband and inter-band relaxation as well as $\mathrm{He}_{2}^{*}$ excimer formation follows the excitation. ${ }^{9,10,13,14}$ Due to the repulsive interaction between excited $\mathrm{He}^{*}$ or $\mathrm{He}_{2}^{*}$ and the He environment the excitation migrates to the surface presumably involving both resonant hopping of the

\footnotetext{
${ }^{a)}$ Current address: IBM-India Semiconductor R\&D Center, D3-F1, Manyata Park, Bangalore 560045, India.

b) mudrich@physik.uni-freiburg.de
}

electronic excitation as well as nuclear motion of the excited He* atom. ${ }^{3,14-16}$ Depending on the size of the He droplet, the $\mathrm{He}^{*}\left(1 \mathrm{~s} 2 \mathrm{p}^{1} \mathrm{P}\right)$ state is trapped at the surface and eventually relaxes into the long-lived $1 \mathrm{~s} 2 \mathrm{~s}^{1,3} \mathrm{~S}$ states or into vibrationally excited $\mathrm{He}_{2}^{*}$ molecules. ${ }^{2}$ The latter are subject to vibrational relaxation by coupling to the He droplet and eventually evaporate off the droplet surface.

(ii) At photon energies $23<h v<24.6 \mathrm{eV}$, the droplet response is even more complex. In addition to the aforementioned relaxation channels, the emission of $\mathrm{He}^{*}$ and $\mathrm{He}_{2}^{*}$ in Rydberg states dominates, ${ }^{10,14,15}$ while the fraction of $\mathrm{He}_{2}^{*}$ dimers increases with rising excitation energies. ${ }^{9}{ }^{9} 10$ At $h v>24 \mathrm{eV}$ population of triplet states of $\mathrm{He}$ was also observed..$^{9,12}$ As a further relaxation channel, autoionization of He droplets sets in at $h v>23 \mathrm{eV}$ leading to the formation of small ionic fragments $\left(\mathrm{He}_{n}^{+}, n \leq 17\right)$ as well as large cluster ions $\left(N \gtrsim 10^{3}\right) .{ }^{6}$ A peculiarity of the ionization of $\mathrm{He}$ droplets below the ionization energy $E_{i, \mathrm{He}}=24.59 \mathrm{eV}$ of atomic $\mathrm{He}$ is the emission of electrons with very low kinetic energy $<1 \mathrm{meV}$ as seen in photoelectron imaging experiments. ${ }^{7,8}$ Recent time-resolved photoelectron and photoion imaging experiments have revealed the dynamics of various relaxation processes in this regime. ${ }^{12-15}$

(iii) At photon energies $h v>24.6 \mathrm{eV}$, that is above $E_{i, \mathrm{He}}$, $\mathrm{He}^{+}$ions (positive holes) are created in the droplets. The positive charges subsequently migrate through the He droplet by resonant hopping and eventually localize 
by forming $\mathrm{He}_{2}^{+}$molecular ions or by ionizing a dopant if present. ${ }^{1,3-5}$ The internal energy of the newly formed ion as well as the binding energy liberated upon formation of "snowball" structures (He atoms tightly bound around the ion core) is believed to stop the chargehopping process and causes massive droplet fragmentation. Therefore, $\mathrm{He}^{+}$largely from background $\mathrm{He}$ atoms and $\mathrm{He}_{2}^{+}$from droplets are the dominant species appearing in the mass spectra. ${ }^{6,8,16,17}$

Detailed insight into the dynamics of photoexcitation and ionization of pure He nanodroplets has been gained from ion mass spectra and velocity-map photoelectron imaging ${ }^{7,8}$ as well as from dispersed fluorescence measurements. ${ }^{9-11,18}$ The photoelectron spectra (PES) recorded by ionizing He droplets at $h v=25 \mathrm{eV}$ have revealed the presence of a high-energy component extending to electron energies $E_{e}>h v-E_{i, \mathrm{He}}$ which was discussed in terms of the direct single ionization of paired up neighboring $\mathrm{He}$ atoms to form $\mathrm{He}_{2}^{+}$dimer ions in bound vibrational levels. ${ }^{8}$ Photoelectron angular distributions (PAD) measured for He droplets were found to be more isotropic than those for free He atoms indicating elastic scattering of the escaping electrons from $\mathrm{He}$ in the droplets. Apart from electrons created by direct photoionization with energies of $h v-E_{i, \mathrm{He}}$ in regime (iii), electrons with nearly vanishing kinetic energy were observed which arise from an indirect ionization mechanism in regime (ii) involving significant electron-He interactions. This component in the PES was most pronounced for large He droplets ionized in regimes (ii) and (iii) up to $h v=27 \mathrm{eV}^{7,8}$ Possible origins such as trapping of electrons in so called bubble states that decay when they approach the droplet surface, ${ }^{19-22}$ or vibrational autoionization of highly excited electronic states of the droplets were discussed. ${ }^{8}$

In the present paper, we report a synchrotron study of pure $\mathrm{He}$ nanodroplets using Velocity-Map Imaging PhotoElectron-PhotoIon COincidence (VMI-PEPICO) spectroscopy. This method allows us to measure PES and PAD in coincidence with specific ion masses which was not possible in previous experiments. The PES and PAD measured in correlation with the most abundant fragments $\mathrm{He}_{n}^{+}, n=1,2,3$ are discussed. The PES and PAD correlating to $\mathrm{He}^{+}$ions from the droplet beam show no significant deviation from those of free $\mathrm{He}$ atoms, indicating that no $\mathrm{He}^{+}$ions are formed by droplet ionization. In contrast, for $\mathrm{He}_{2}^{+}$and $\mathrm{He}_{3}^{+}$we find slightly altered PES as compared to $\mathrm{He}^{+}$as well as reduced anisotropy of the PADs, presumably due to elastic scattering of the outgoing photoelectrons with $\mathrm{He}$ atoms in the droplets. This effect was previously observed with rare gas clusters $\left(\mathrm{Ar}^{23,24} \mathrm{Kr},{ }^{23}\right.$ and $\left.\mathrm{Xe}^{23,25,26}\right)$ even for mean cluster sizes as small as $N \lesssim$ 100. We find indications for multiple excitations and subsequent decay by Penning-like ionization when irradiating the droplets at $h v=21.6 \mathrm{eV}$ which corresponds to the maximum of the $1 \mathrm{~s}^{2}{ }^{1} \mathrm{~S} \rightarrow 1 \mathrm{~s} 2 \mathrm{p}^{1} \mathrm{P}$ droplet absorption band. ${ }^{6,18,27}$ Upon ionization of $\mathrm{He}$ droplets at high photon energies $h v \gtrsim 2$ $\times E_{i, \mathrm{He}}$ we observe low-energy electrons in addition to those directly emitted, which are generated by inelastic electron-He collisions akin to excitonic satellites observed previously in PES of $\mathrm{Ne}$ and Ar clusters. ${ }^{28}$

\section{EXPERIMENTAL}

The experiments presented here are performed using a mobile He droplet machine attached to a VMI-PEPICO detector at the GasPhase beamline of Elettra-Sincrotrone Trieste, Italy. ${ }^{29}$ The experimental setup is described in more detail in a previous publication. ${ }^{16}$ In short, a continuous beam of $\mathrm{He}$ nanodroplets with a mean size ranging from 200 to $17000 \mathrm{He}$ atoms per droplet is generated by varying the temperature $T_{0}$ of a cryogenic nozzle. ${ }^{30,31}$ An adjacent vacuum chamber contains doping cells, which are not used in the experiments reported here, and a mechanical beam chopper for discriminating ion and electron counts correlated with the droplet beam from background counts due to residual $\mathrm{He}$ and other residual gas components.

In the detector chamber further downstream, the $\mathrm{He}$ droplet beam intersects the synchrotron light beam at right angles in the center of a velocity map imaging (VMI) spectrometer. The synchrotron radiation is linearly polarized along the direction of the He droplet beam, which is perpendicular to the symmetry axis of the VMI spectrometer. The latter is composed of field plates that accelerate photoelectrons onto a position and time resolving delay-line detector, while photoions are accelerated onto a microchannel plate detector without position resolution just to record flight times. Measuring electrons and ions in coincidence allows us to extract from the data both ion mass spectra and mass-correlated velocity-map photoelectron images. The latter are transformed into PES and PAD using standard Abel inversion programs. ${ }^{32,33}$

The narrow-band synchrotron radiation $\left(E / \Delta E \gtrsim 10^{4}\right)$ is varied between 21 and $66 \mathrm{eV}$ in this study. All photon energy dependent ion and electron spectra are normalized to the light intensity which is monitored by a calibrated photodiode. Note that a non-negligible amount of second- and third-order radiation is present at the lower end of the tuning range $h v \lesssim 20 \mathrm{eV}$. The pulse repetition rate is $500 \mathrm{MHz}$ and the peak intensity in the interaction region is estimated to $I \sim 15 \mathrm{~W} \mathrm{~m}^{-2}$.

An additional beam-dump chamber is attached to the end of the apparatus which contains a channel electron multiplier mounted directly in the path of the He droplet beam. It is used for measuring the yield of metastable He atoms and droplets excited by the synchrotron radiation.

\section{RESULTS AND DISCUSSION}

In this work, we focus on ion-mass correlated photoelectron spectroscopy of pure He nanodroplets irradiated by EUV radiation at variable photon energies $h v<E_{i, \mathrm{He}}$ up to $h v \gtrsim 2$ $\times E_{i, \mathrm{He}}$. Let us start the discussion of experimental results by presenting typical ion mass spectra. The dependence of electron and ion yields on the experimental parameters (photon energy $h v$, He droplet size $N$ ) will be discussed subsequently. Finally, the ion-mass correlated PES and PAD at variable $h v$ will be presented.

\section{A. Ion yield spectra}

Figure 1 compares typical mass spectra recorded at $h v$ $=25 \mathrm{eV}$ (a) and at $h v=23.8 \mathrm{eV}$ (b). The use of a mechanical 


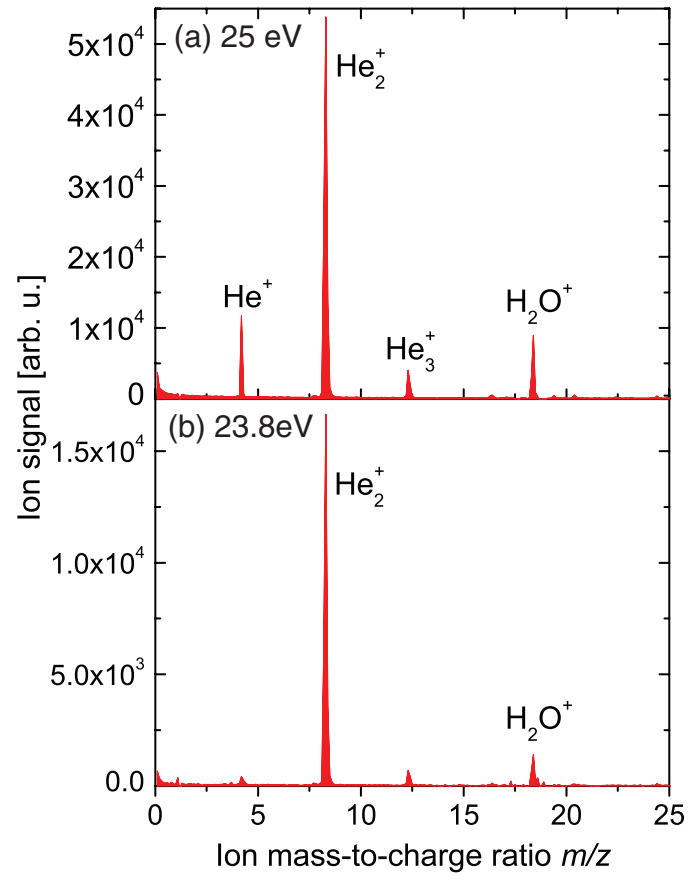

FIG. 1. Difference mass spectra of ionized (a) and autoionizing (b) He nanodroplets. The He expansion conditions are $p_{0}=50$ bars and $T_{0}=23 \mathrm{~K}(\mathrm{~N}$ $=1900)$.

chopper that periodically intercepts the He droplet beam allows us to discriminate the ion signals originating from the He droplet beam from background gas ions. When the beam chopper is in the "open" position, both contributions are measured whereas in the "closed" position, only background ions contribute. Thus, the shown difference signal visualizes the contribution correlated to the He droplet beam only. At $h v$ $=25 \mathrm{eV}$ (a) the He atoms in the droplets are directly ionized (regime (iii)), whereas at $h v=23.8 \mathrm{eV}$ (b) the He droplets are resonantly excited into the droplet equivalent of the $1 \mathrm{~s} 3 \mathrm{p}^{1} \mathrm{P}$ and $1 \mathrm{~s} 4 \mathrm{p}^{1} \mathrm{P}$ atomic He levels out of which they decay by autoionization and other processes (regime (ii)). The He droplet beam source is operated at $\mathrm{He}$ expansion conditions of $p_{0}$ $=50$ bars and $T_{0}=23 \mathrm{~K}$. The corresponding mean He droplet size amounts to $N=1900 .^{31}$

At photon energies $h v>E_{i, \mathrm{He}}$ (Fig. 1(a)), the highest intensity mass peaks in the spectra are those of $\mathrm{He}^{+}$and $\mathrm{He}_{2}^{+}$. Note that $\mathrm{He}_{2}^{+}$is even more abundant than $\mathrm{He}^{+}$, in contrast to earlier electron impact and synchrotron experiments. ${ }^{2,6,17,34}$ This may be due to a better suppression of background contributions in the present experiment by (a) chopping the droplet beam and (b) providing a long flight distance from the nozzle up to the ionization region of $71 \mathrm{~cm}$, which results in a highly collimated droplet beam where the content of free $\mathrm{He}$ atoms accompanying the droplet beam is further diminished. The efficient formation of $\mathrm{He}_{2}^{+}$ions agrees with the established notion that the initially created $\mathrm{He}^{+}$positive hole migrates within the $\mathrm{He}$ droplets and localizes by forming a $\mathrm{He}_{2}^{+}$ion. The binding energy liberated by forming the $\mathrm{He}_{2}^{+}$molecule as well as by forming a tightly bound shell of $\mathrm{He}$ atoms around the ion ("snowball") subsequently induces droplet fragmentation and the ejection of bare $\mathrm{He}_{2}^{+}$. Higher $\mathrm{He}_{n}^{+}$cluster ion masses are also present with lower

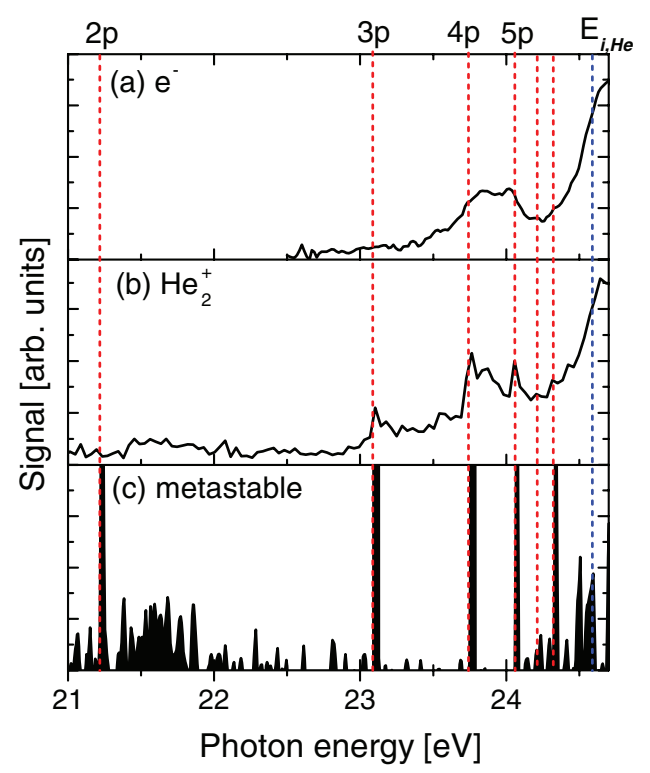

FIG. 2. Photon energy dependence of the yield of photoelectrons (a), $\mathrm{He}_{2}^{+}$ ions (b) and metastable atoms and droplets (c). The vertical dashed lines indicate $\mathrm{He}$ atomic level energies.

intensities in the entire mass range shown. The $\mathrm{H}_{2} \mathrm{O}^{+}$signal stems from water molecules picked up by the He droplets from the residual gas which are ionized by charge transfer ionization.

At photon energies $h v<E_{i, \mathrm{He}}$ (Fig. 1(b)), the $\mathrm{He}^{+}$signal nearly completely vanishes as expected due to energy conservation. Here, however, at $h v=23.8 \mathrm{eV}$, that is in regime (ii), aside from other relaxation channels the excited He droplets are subject to autoionization yielding $\mathrm{He}_{2}^{+}$and small $\mathrm{He}_{n}^{+}$ cluster ions as well as ultraslow photoelectrons. ${ }^{6,9,13,15}$ This explains the high $\mathrm{He}_{2}^{+}$yield as compared to all other masses.

The dependence of the characteristic ionization signals on the photon energy $h v$ is studied by recording the noncoincident electron and $\mathrm{He}_{2}^{+}$ion signals for varying $h v$. The resulting spectra are depicted in Fig. 2. The He expansion conditions are set to $p_{0}=50$ bars and $T_{0}=21 \mathrm{~K}$ corresponding to a mean droplet size of $N=2900$ for all photon energies. Different vertical scales are used for the three panels (a)-(c). The yield of metastable atoms and droplets shown in (c) is recorded in the "chopper open" position using a single channel electron multiplier mounted such that the entire $\mathrm{He}$ droplet beam hits the detector. Therefore, it contains contributions from both, the He droplet beam as well as from atomic He effusing into the detection chamber. Note that we cannot strictly exclude contributions to the signal from EUV fluorescence light reaching the electron multiplier.

The broadband structure in Figs. 2(a) and 2(b) at photon energies $23 \leq h v \leq 24.6 \mathrm{eV}$ is in good agreement with previous ionization spectra recorded with neat He nanodroplets. ${ }^{6}$ Note that we systematically measure higher electron count rates than total ion yields by a factor of 5-15 depending on $h v$. This indicates the partial presence of large $\mathrm{He}_{n}^{+}$cluster ions with $n>100$ which fall beyond the detection range of our setup. ${ }^{6}$ The peaked structures around 21.8, 23.1, 23.8 and $24.7 \mathrm{eV}$ in (a) and (b) can be assigned to excited He droplet 
states that mostly derive from the $1 \mathrm{~s} 2 \mathrm{p}^{1} \mathrm{P}, 1 \mathrm{~s} 3 \mathrm{p}^{1} \mathrm{P}, 1 \mathrm{~s} 4 \mathrm{p}^{1} \mathrm{P}$, and highly excited Rydberg levels of atomic He. At $h v>24.6 \mathrm{eV}$, the He droplets are directly ionized yielding the highest ion and electron signals. When varying the mean droplet size by changing the He nozzle temperature between $T_{0}=17$ and $27 \mathrm{~K}$ the overall $\mathrm{He}_{2}^{+}$count rate slightly changes with a maximum at $T_{0}=21 \mathrm{~K}$ but the structure of the spectrum remains nearly constant. Surprisingly, we find a weak broad maximum in the $\mathrm{He}_{2}^{+}$signal around $h v=21.6 \mathrm{eV}$ which corresponds to the $1 \mathrm{~s} 2 \mathrm{p}^{1} \mathrm{P}$ excitation band of He droplets. Since this band lies below $E_{i, \mathrm{He}}$ by about $3 \mathrm{eV}$, which is more than the binding energy of $\mathrm{He}_{2}^{+}$, autoionization of singly excited droplets is impossible. As we will discuss below, we attribute this feature to multiply excited He droplets that decay by a Penning-like process in which one $\mathrm{He}^{*}$ excitation relaxes to the ground state whereas the other $\mathrm{He}^{*}$ is ionized.

The signal measured using the ion detector intercepting the droplet beam at the end of the beam line shows sharp peaks corresponding to atomic lines as well as one broad maximum around $h v=21.6 \mathrm{eV}$ (Fig. 2(c)). The sharp atomic lines at energies corresponding to excitation of the levels $1 \operatorname{snp}^{1} \mathrm{P}$, $\mathrm{n}=2,3, \ldots$ reflect the detection of metastable atomic states of He populated by radiative decay of the ${ }^{1} \mathrm{P}$ states excited in the atomic He part of the beam. ${ }^{35}$ We attribute the broad peak at $h v=21.6 \mathrm{eV}$ to the $1 \mathrm{~s} 2 \mathrm{p}^{1} \mathrm{P}$ droplet excitation which decays by relaxation into the metastable $1 \mathrm{~s} 2 \mathrm{~s}^{1} \mathrm{~S}$ state of $\mathrm{He}$ atoms or into the lowest ${ }^{1} \Sigma_{u, g}^{+}$state of $\mathrm{He}_{2}^{*}$ excited dimers. The latter either remain weakly bound to the droplet surface or desorb off the droplets due to vibrational relaxation. ${ }^{2}$ Note that the relaxation of the $1 \mathrm{~s} 2 \mathrm{p}^{1} \mathrm{P}$ droplet excitation into $1 \mathrm{~s} 2 \mathrm{~s}^{1} \mathrm{~S}$ and even lower-lying levels of $\mathrm{He}^{*}$ and $\mathrm{He}_{2}^{*}$ was previously observed for doped droplets. ${ }^{16,36}$

\section{B. Photoelectron imaging}

In order to obtain more detailed information about the dynamics of He droplet ionization in the different regimes (i)(iii) we record photoelectron images in coincidence with the most abundant ions $\mathrm{He}^{+}$and $\mathrm{He}_{2}^{+}$. Figure 3 gives an overview of such images recorded at various photon energies $h v$. In these images, the polarization vector of the synchrotron radiation is oriented vertically in the image plane as indicated by the arrow in (a). The electron distribution measured in coincidence with $\mathrm{He}^{+}$at $h v=25 \mathrm{eV}$ (a) shows an anisotropic ring-shaped structure which matches the characteristic angular distribution of a p-wave, as expected for direct one-photon ionization out of the He 1s-orbital. The electrons correlating to $\mathrm{He}_{2}^{+}$emitted at the same photon energy (b) feature a similar ring-shaped distribution which has the same radius but is more isotropic. The PAD of directly emitted electrons recorded in correlation with $\mathrm{He}_{2}^{+}$and $\mathrm{He}_{3}^{+}$are analyzed further below.

At the photon energy $h v=24 \mathrm{eV}$, that is in regime (ii), the electron signal correlating to $\mathrm{He}^{+}$nearly vanishes, whereas that correlating to $\mathrm{He}_{2}^{+}$concentrates in a small central spot indicating very low electron kinetic energy. As mentioned above, nearly zero kinetic energy electrons have been observed in many experiments with pure and doped $\mathrm{He}$ droplets. $^{7,8,13,16,36,37}$ They appear most prominently when $h v$
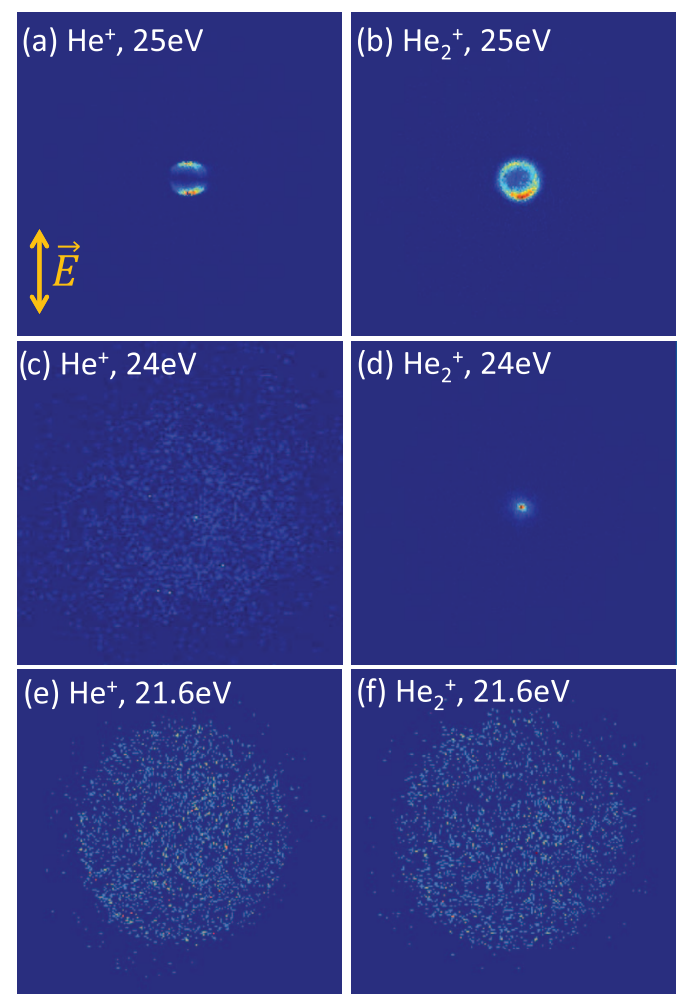

FIG. 3. Raw velocity map images of photoelectrons from He droplets in correlation with $\mathrm{He}^{+}$(a), (c), (e) and with $\mathrm{He}_{2}^{+}$(b), (d), (f) irradiated at photon energies $h v=21.6,24,25 \mathrm{eV}$. The arrow in (a) indicates the direction of the polarization vector of the EUV radiation.

is tuned slightly below $E_{i, \mathrm{He}}$ and droplet autoionization becomes an important decay channel.

Surprisingly, at $h v=21.6 \mathrm{eV}$, that is in regime (i) of pure droplet excitation into the $1 \mathrm{~s} 2 \mathrm{p}^{1} \mathrm{P}$ band, significant electron signals correlating to both $\mathrm{He}^{+}$and $\mathrm{He}_{2}^{+}$are recovered. The two images feature extended isotropic circular structures of nearly equal size. As discussed below in more detail, we attribute these electrons to the decay of multiply excited $\mathrm{He}$ droplets by Penning-like ionization.

\section{Mass-correlated photoelectron spectra}

First, we examine the PES which we obtain by inverse Abel transformation and angular integration of the photoelectron images recorded in regime (iii). The spectra shown in Fig. 4 are recorded at $h v=25 \mathrm{eV}$ for different He droplet sizes by varying the nozzle temperature $T_{0}$ as indicated in the legend. The spectra correlating to $\mathrm{He}^{+}$(Fig. 4(a)) are obtained from the background-subtracted ("chopper closed") electron images of the full signal ("chopper open") so as to discriminate the electrons correlated with the He droplet beam. The dashed line represents the result of fitting the average of the experimental curves by a log-normal distribution function, which is an empirical function that reproduces well the measured peak shape. The maximum is peaked at $0.39(2) \mathrm{eV}$ which matches the excess energy $h v-E_{i, \mathrm{He}}$ $=0.41 \mathrm{eV}$ in the direct ionization of He atoms. The width of the spectral feature of $\mathrm{He}^{+}$reflects the energy resolution of the spectrometer and matches the width measured for atomic $\mathrm{He}$ from background gas. Thus, within the experimental 


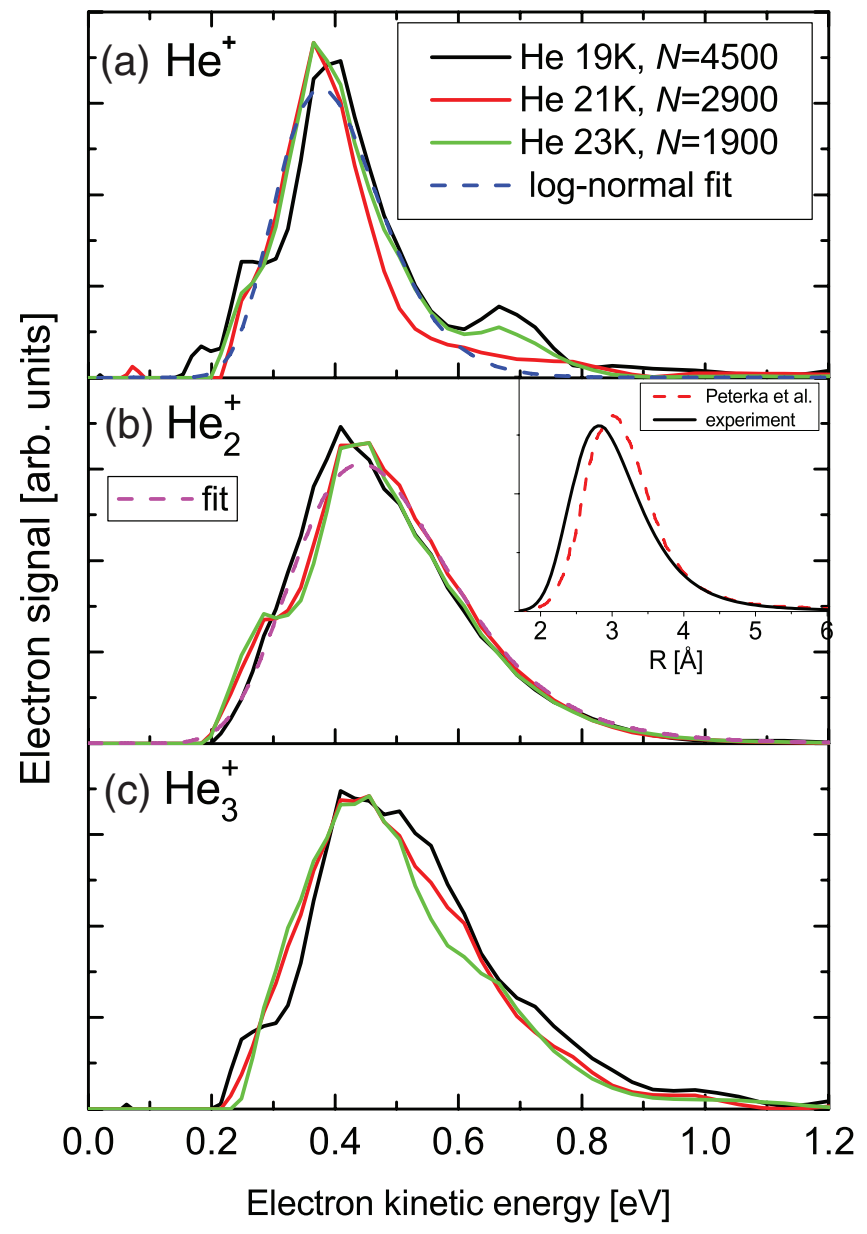

FIG. 4. Photoelectron spectra measured in coincidence with $\mathrm{He}^{+}$(a), $\mathrm{He}_{2}^{+}$ (b), and $\mathrm{He}_{3}^{+}$ions (c) at $h v=25 \mathrm{eV}$ for different $\mathrm{He}$ droplet sizes. The $\mathrm{He}^{+}$spectrum (a) is modeled by a log-normal distribution (dashed line). The dashed line in (b) represents the convolution of the fit function in (a) and a log-normal distribution that accounts for the pure $\mathrm{He}_{2}^{+}$spectrum. The inset in (b) compares the resulting nearest-neighbor distribution for pairs of He atoms with an ab initio calculation by Peterka et al. ${ }^{8}$

uncertainties, we see no significant influence of the presence of He droplets on the PES. This suggests that the $\mathrm{He}^{+}$atomic ions stem from the atomic component that accompanies the droplet beam. Possibly, however, there is a contribution from $\mathrm{He}$ atoms located at the outer surface of the droplets where the He density is too low to cause significant line shifts and to induce charge migration and $\mathrm{He}_{2}^{+}$formation. ${ }^{5}$ As discussed in Sec. III B 2, the high degree of anisotropy of the $\mathrm{He}^{+}$correlated photoelectrons (Figs. 5 and 6) further supports this interpretation.

The peak in the PES correlating to $\mathrm{He}_{2}^{+}$(Fig. 4(b)), however, is slightly shifted and significantly broadened towards higher kinetic energies. The spectrum of $\mathrm{He}_{3}^{+}$(c) is even more extended towards higher electron energies than that of $\mathrm{He}_{2}^{+}$. In previous work, Peterka et al. ${ }^{8}$ have measured and analyzed the total PES recorded without ion mass correlation. The presence of a shoulder extending to higher energies was interpreted using a simple model based on the assumption that ionization occurs vertically for pairs of closely spaced $\mathrm{He}$ atoms in the droplet, thereby accessing the attractive potential region of the cationic $\mathrm{He}_{2}^{+}$core. The resulting photoelectrons have higher kinetic energy than those of atomic He. ${ }^{8}$ The shape of
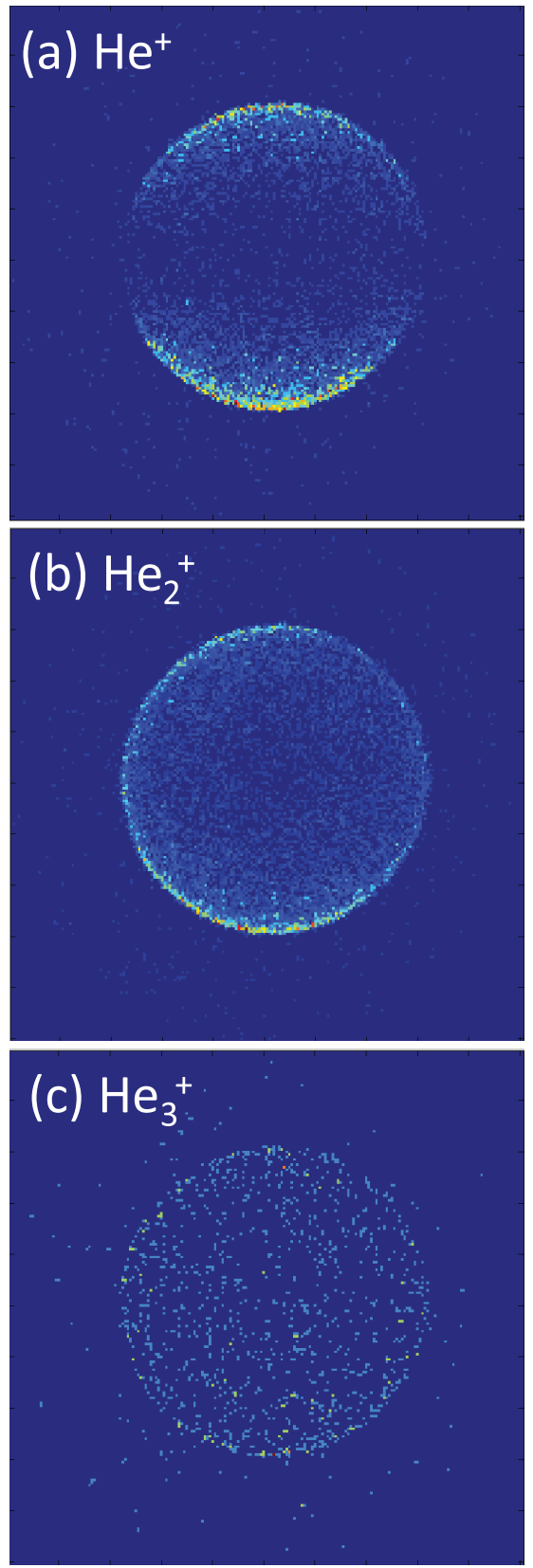

FIG. 5. Raw velocity map images of photoelectrons from ionized He droplets at $h v=35 \mathrm{eV}$ recorded in coincidence with $\mathrm{He}^{+}$(a), $\mathrm{He}_{2}^{+}$(b), and $\mathrm{He}_{3}^{+}$(c) ions.

this shoulder was simulated by a Franck-Condon model based on the $\mathrm{He}_{2}$ and $\mathrm{He}_{2}^{+}$difference potential and the distribution of $\mathrm{He}-\mathrm{He}$ interatomic distances for the nearest $\mathrm{He}-\mathrm{He}$ atom pairs ("nearest neighbor") obtained from path integral Monte Carlo calculations.

We adopt the same model in order to infer the distribution $n n(r)$ of distances $r$ for nearest $\mathrm{He}-\mathrm{He}$ atom pairs which we identify as precursors for $\mathrm{He}_{2}^{+}$formation. Note that this model of vertical transitions from $\mathrm{He}-\mathrm{He}$ pairs into bound levels of $\mathrm{He}_{2}^{+}$does not contradict the concept of creating $\mathrm{He}^{+}$ charges that migrate through the droplets before localizing by forming deeply bound $\mathrm{He}_{2}^{+}$. Due to the presence of one or more additional $\mathrm{He}$ atoms close to the respective $\mathrm{He}-\mathrm{He}$ pair the total interaction potential is extended to a double-well or 

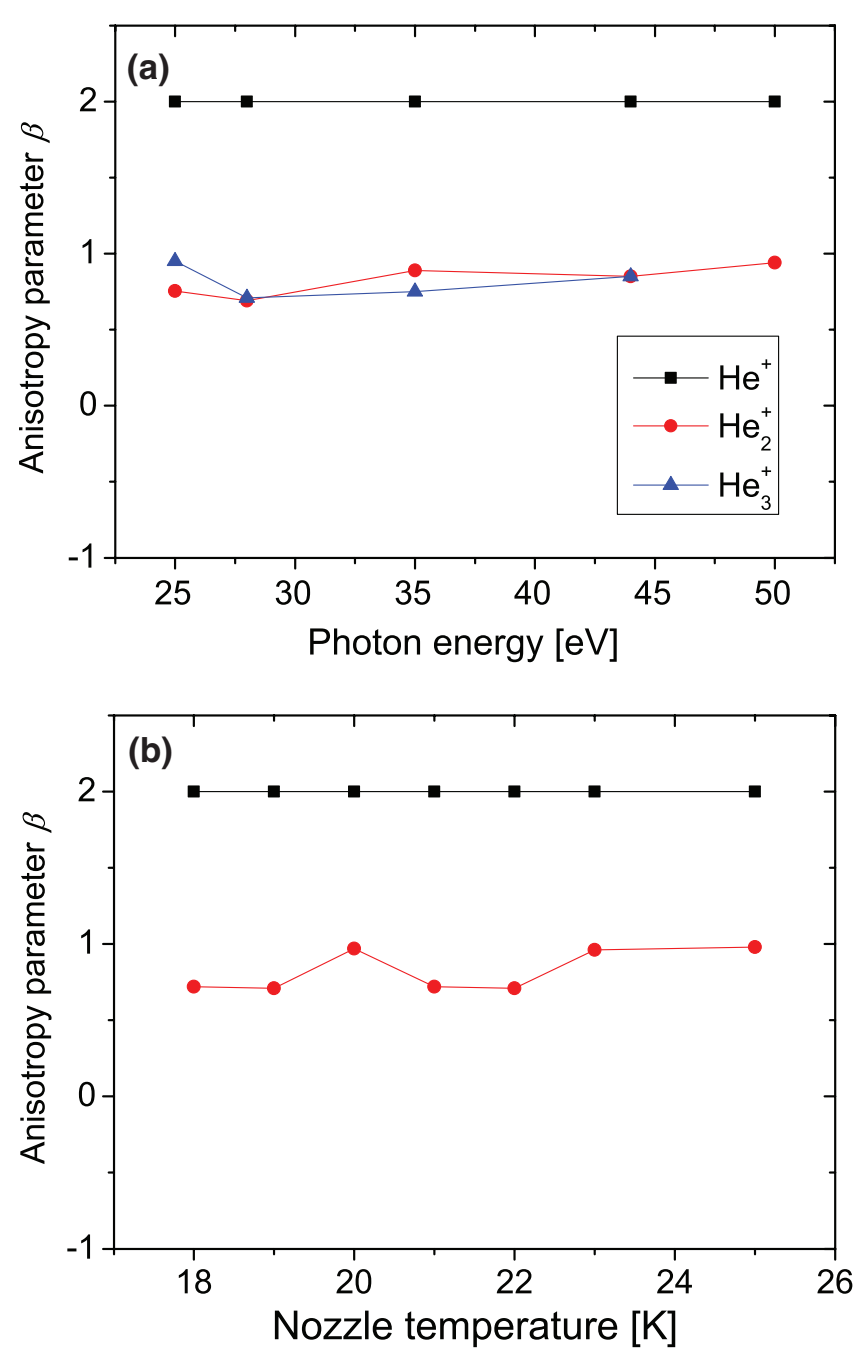

FIG. 6. Anisotropy parameters $\beta$ inferred from the photoelectron images correlating to $\mathrm{He}^{+}, \mathrm{He}_{2}^{+}$, and $\mathrm{He}_{3}^{+}$ions as a function of $h v$ (a) and as a function of nozzle temperature in the range $T_{0}=18-25 \mathrm{~K}$ corresponding to $N=5600-1200$ (b). In (a) $N=4500$ and in (b) $h v=25 \mathrm{eV}$.

multiple-well potential where the heights of the barriers depend on the distances between the three or more atoms. For sufficiently close spacing and excitation of high-lying levels above the barriers, the charge is therefore delocalized. Localization then occurs due to vibrational relaxation into one potential well which prevents further hopping over or tunneling through a barrier. Thus, the low-energy edge of the $\mathrm{He}_{2}^{+}$peak (Fig. 4(b)) is associated with $\mathrm{He}_{2}^{+}$formed after charge migration, whereas the high-energy tail is identified with direct formation of deeply bound $\mathrm{He}_{2}^{+}$.

The model curve shown as a dashed line in Fig. 4(b) is obtained by fitting the convolution of the fit function of the $\mathrm{He}^{+}$peak (dashed line Fig. 4(a)) with a log-normal distribution function $f_{\mathrm{He}_{2}^{+}}\left(E_{e}\right)$. This function is chosen empirically to describe the characteristic line shape of the $\mathrm{He}_{2}^{+}$component. From that distribution we obtain $n n(r)$ by mapping the energy distribution $f_{\mathrm{He}_{2}^{+}}$onto the shifted difference potential $\Delta V=V_{\mathrm{He}_{2}^{+}}-V_{\mathrm{He}_{2}}+h v-E_{i, \mathrm{He}}{ }^{38,39}$ using the transformation $n n(r)=f_{\mathrm{He}_{2}^{+}}(\Delta V(r)) d \Delta V(r) / d r$ where $h v=25 \mathrm{eV}$. The result is shown as a solid line in the inset of Fig. 4(b). It only slightly deviates from the original calculation (dashed line) in that its maximum is slightly shifted to a shorter $\mathrm{He}-\mathrm{He}$ distance $r=2.9 \AA$ instead of $R=3.0 \AA$ in the original calculation. Since the measured $\mathrm{He}_{2}^{+}$ions are formed in regions of varying density inside or at the surface of the droplets the shown $n n(r)$ distribution is a density average for the present experimental conditions.

\section{Mass-correlated photoelectron angular distributions}

Next, we discuss the angular distributions of photoelectrons correlating to $\mathrm{He}^{+}, \mathrm{He}_{2}^{+}$, and $\mathrm{He}_{3}^{+}$in more detail. To this end, we record photoelectron images at variable $h v$ up to $50 \mathrm{eV}$ for droplet sizes ranging from $N=1200$ to 5600. Typical raw photoelectron images recorded at $h v=35 \mathrm{eV}$ are depicted in Fig. 5. As for $h v=25 \mathrm{eV}$ (Figs. 3(a) and 3(b)), we note a reduced anisotropy of the photoelectron distributions correlating to the molecular ions $\mathrm{He}_{2}^{+}$and $\mathrm{He}_{3}^{+}$(Figs. 5(b) and 5(c)). From the images, we infer the average anisotropy parameter $\beta$ by fitting the angular dependence of the signal intensity $I(\theta)$ in the inverse Abel transformed images using the standard expression $I(\theta) \propto 1+\beta P_{2}(\cos \theta){ }^{40}$

The resulting values of $\beta$ are compiled in Fig. 6(a) for variable $h v$ and in Fig. 6(b) for variable $T_{0}$ (droplet size). While for electrons correlating to $\mathrm{He}^{+}$we find a constant value $\beta=2.0(1)$ as expected for the direct ionization of unperturbed $\mathrm{He}$ atoms, for $\mathrm{He}_{2}^{+}$and $\mathrm{He}_{3}^{+}$the anisotropy parameter is reduced to $\beta=0.8(1)$ which does not significantly vary as a function of the parameters $h v$ and $T_{0}$. On the one hand, this seems to indicate that $\mathrm{He}_{2}^{+}$and $\mathrm{He}_{3}^{+}$also merely stem from the $\mathrm{He}_{2}$ and $\mathrm{He}_{3}$ molecular components which accompany the He droplet beam. On the other hand, the fact that the photoelectron distributions measured in coincidence with dopant ions generated by charge transfer ionization ${ }^{16}$ strongly resemble those of $\mathrm{He}_{2}^{+}$suggests that $\mathrm{He}_{2}^{+}$do stem from droplets. Besides, the extremely weak perturbation of the $\mathrm{He}$ atomic orbital by the binding of $\mathrm{He}$ in $\mathrm{He}_{2}$ or $\mathrm{He}_{3}$ van der Waals molecules is not likely to cause substantial deviations of the PAD from that of atomic He. The reduced anisotropy is probably due to elastic scattering of the outgoing photoelectron from the He droplets. We believe that the probed range of droplet sizes is not sufficiently broad to see a significant influence of a changing average He density on the photoelectron distribution. Note that the first observation of the reduction of the anisotropy of the PADs from Xe clusters did not exhibit a size dependence. ${ }^{25}$ In that work, for Xe clusters in the size range $N=1000-4000$, the observed anisotropy parameter $\beta$ was reduced by factors 0.6 and 0.9 for photoelectrons coming from cluster bulk and surface atoms, respectively, which compares reasonably well with the anisotropy reduction of 0.4 measured in this work for He nanodroplets. A significant size-dependent anisotropy reduction due to elastic scattering was only observed for rare gas clusters at low photon energies $\lesssim 100 \mathrm{eV}$ and for large cluster sizes $N>1000 .^{23,26}$ A better understanding of the PAD and spectra requires further experimental and theoretical efforts. In particular, the comparison with PES of massselected $\mathrm{He}_{2}$ as recently studied ${ }^{41}$ would give interesting new insights into the effect of the He droplet on the photoelectron distributions. 

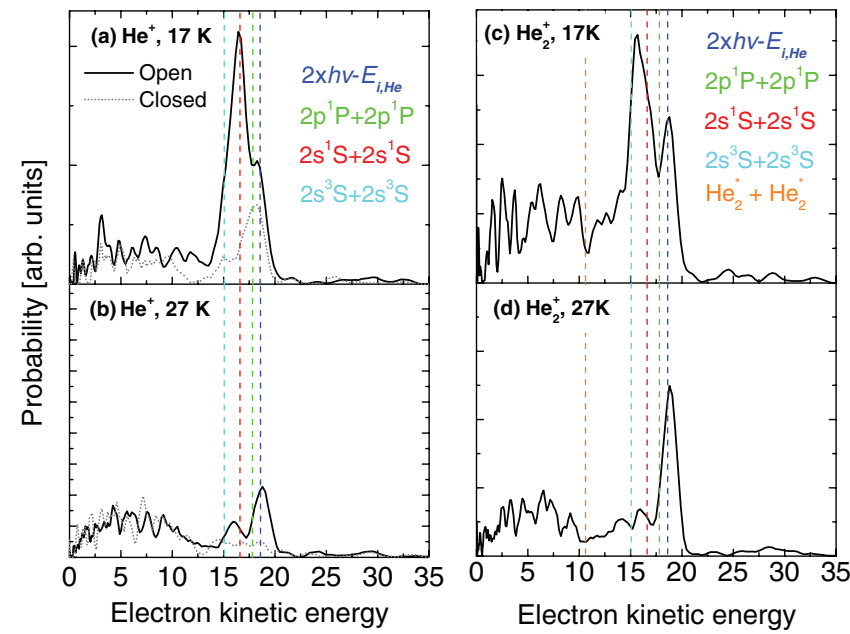

FIG. 7. Photoelectron spectra measured in coincidence with $\mathrm{He}^{+}$(a), (b) and with $\mathrm{He}_{2}^{+}$ions (c), (d) at $h v=21.6 \mathrm{eV}$ and at expansion conditions $p_{0}$ $=50$ bars and $T_{0}=17 \mathrm{~K}(N=7000)(\mathrm{a}),(\mathrm{c})$ and $T_{0}=27 \mathrm{~K}(N=800)(\mathrm{b})$, (d). The vertical lines indicate electron energies expected for the relaxation of two excited He atoms into various 1s2p-levels or into the lowest excited state of $\mathrm{He}_{2}^{+}$. Labels "Open" and "Closed" refer to data recorded in the open and closed positions of the droplet beam chopper.

\section{Penning ionization}

So far, we have examined the photoelectron distributions of $\mathrm{He}$ nanodroplets in the regime (iii) of direct ionization. However, the photon energy dependent ion yield measurements (Fig. 2) and photoelectron images (Fig. 3) have revealed weak ionization signals even at $h v=21.6 \mathrm{eV}$ which falls below the droplet autoionization threshold.

The PES recorded in coincidence with $\mathrm{He}^{+}$and $\mathrm{He}_{2}^{+}$ are depicted in Fig. 7 for two different He droplet sizes $N$ $=7000$ ((a) and (c)) and $N=800$ ((b) and (d)). In all measurements we distinguish 3 components in the PES. A sharp peak at electron energies $E_{e}=2 \times h v-E_{i, \mathrm{He}}=18.6 \mathrm{eV}$ results from direct $\mathrm{He}$ ionization by second-order radiation and possibly from electrons emitted by third-order radiation which have undergone an inelastic collision with surrounding $\mathrm{He}$ atoms, as discussed in the last part of this section. This peak is the dominant feature in the spectra recorded for small He droplets $N=800$ (Figs. 7(b) and 7(d)). In addition, the spectra recorded for large droplets (Figs. 7(a) and 7(c)) exhibit a pronounced peak shifted to lower electron energies $E_{e} \approx$ $16 \mathrm{eV}$. The broad structure at low energies around $E_{e}=7 \mathrm{eV}$, which is also present in the spectra from the effusive background, is attributed to background signal presumably from scattered photons and from false coincidences.

These spectra closely resemble the ones measured in coincidence with rare-gas or alkali metal dopant ions. ${ }^{16,36}$ In those experiments, the peak structures, similar to the ones visible in Fig. 7 between $h v=15$ and $20 \mathrm{eV}$, were assigned to electrons produced by ionization of dopants $X$ in a Penninglike process

$$
\mathrm{He}^{*}\left[\mathrm{He}_{N}\right]+X \rightarrow \mathrm{He}\left[\mathrm{He}_{N}\right]+X^{+}+e^{-},
$$

where the droplet-induced relaxation of $\mathrm{He}^{*}$ into lowerlying levels such as $1 \mathrm{~s} 2 \mathrm{~s}^{1} \mathrm{~S}$ was likely to precede Penning ionization. ${ }^{16,36}$ In the present case of $\mathrm{He}^{+}$correlated PES, we take the presence of components shifted to lower energies $E_{e}$ $<2 \times h v-E_{i, \mathrm{He}}$ as an indication for a Penning-like reaction involving two $\mathrm{He}^{*}$ excitations in the same droplet as given by Eq. (1), for $X=\mathrm{He}^{*}$. This process has been discussed in the context of reduced EUV fluorescence emission observed when resonantly exciting large He droplets $\left(N>10^{4}\right) .{ }^{11}$

The probability for double excitation of a He nanodroplet $P_{2}=P_{1}^{2}$ by the interaction with one synchrotron pulse can be estimated from the probability of single excitation, $P_{1}=N \sigma_{a} \Delta t I /(e h v) \sim 3 \times 10^{-9}$, where $I$ is the light intensity. Here, we assume an absorption cross section $\sigma_{a} \approx 25 \mathrm{Mb}$ of one He atom in a droplet containing $N$ atoms, $\Delta t \approx 130 \mathrm{ps}$ is the pulse length, and $e$ and $h$ denote the elementary charge and Planck's constant, respectively. When assuming that a fraction of excited He droplets relaxes into metastable states with life times $\tau$ exceeding the pulse repetition period $T$ $=2 \mathrm{~ns}, P_{1}$ is replaced by $P_{1} t_{t r} / T \approx 500 P_{1}$, where $t_{t r} \approx 1 \mu \mathrm{s}$ denotes the transit time of the droplets through the focus of the synchrotron beam. For those droplets we obtain $P_{2} \approx 3 \times 10^{-12}$, which yields a signal count rate $S$ $=P_{2} N_{\mathrm{HeN}} / t_{t r} \sim 0.5 \mathrm{~s}^{-1}$. Here, the number of He droplets in the focal volume $N_{\mathrm{HeN}}$ amounts to $N_{\mathrm{HeN}}=n_{\mathrm{HeN}} d w^{2}$ $\approx 6 \times 10^{4}$, where $n_{\mathrm{HeN}}=10^{8} \mathrm{~cm}^{-3}$ stands for the number density of He droplets, $d=4 \mathrm{~mm}$ is the diameter of the droplet beam, and $w=400 \mu \mathrm{m}$ is the focus diameter. This estimate approximately matches the count rate measured experimentally. The rapid increase of the Penning signal with increasing droplet size $N$ can be rationalized by the quadratic dependence $S \sim N^{2}$. Note, however, that this estimate relies on the population of metastable excitations which leads to an accumulation of excitations in one droplet over many light pulses. In contrast to that, He droplets that are multiply excited by single intense ultrashort light pulses as available from free-electron lasers will autoionize by a different mechanism akin to interatomic Coulombic decay. ${ }^{42,43}$

Thus, the Penning ionization process appears to be very efficient relative to the decay of $\mathrm{He}^{*}$ or $\mathrm{He}_{2}^{*}$ excitations by spontaneous relaxation or by desorption off the droplet surface. This interpretation is supported by the results of earlier experiments studying the dynamics of excitations in bulk superfluid $\mathrm{He}$ and on molecular beam studies of $\mathrm{He}^{*}-\mathrm{He}^{*}$ Penning collisions. In superfluid He the lifetimes of $\mathrm{He}^{*}$ in $1 \mathrm{~s} 2 \mathrm{~s}^{3} \mathrm{~S}$ and of $\mathrm{He}_{2}^{*}$ excimers in their lowest state $a^{3} \Sigma_{u}^{+}(v=0)$ were measured to be about $15 \mu \mathrm{s}$ and $13 \mathrm{~s}$, respectively. ${ }^{44,45}$ However, upon producing multiple excitations in bulk $\mathrm{He}$ the $\mathrm{He}_{2}^{*}$ excimer population was found to decay due to binary Penning ionization collisions with a rate coefficient rising up to $2 \times 10^{-10} \mathrm{~cm}^{3} / \mathrm{s}$ at He temperatures $1.5 \mathrm{~K}^{44,45}$ Translating this to a He nanodroplet of radius $50 \AA$ containing 12000 $\mathrm{He}$ atoms and two $\mathrm{He}_{2}^{*}$ excimers this corresponds to a decay time of about $1.3 \mathrm{~ns}$, provided the $\mathrm{He}_{2}^{*}$ move freely inside the $\mathrm{He}$ droplets as in superfluid He. Note that the crossover from diffusive to ballistic motion of $\mathrm{He}_{2}^{*}$ in superfluid $\mathrm{He}$ was observed only recently in the temperature range between 100 and $200 \mathrm{mK}$, close to the He droplet temperature. ${ }^{46}$ The Penning collision rate for $\mathrm{He}^{*}$ can be estimated using the known cross section $\sigma_{\mathrm{He}^{*}} \approx 300 \AA^{2}$ from molecular beam scattering experiments. ${ }^{47}$ Assuming a mean relative velocity of the $\mathrm{He}^{*}$ atoms of $60 \mathrm{~m} / \mathrm{s}$ the rate coefficient amounts to about 
$1.8 \times 10^{-10} \mathrm{~cm}^{3} / \mathrm{s}$ and the Penning collision time is $1.5 \mathrm{~ns}$. Thus, from these considerations we may expect that Penning ionization occurs within one synchrotron pulse repetition period and inside the interaction region of the droplet beam with the EUV beam. Note that large Penning ionization rates were also observed for He droplets doped with alkali atoms which reside in weakly bound states at the droplet surface. ${ }^{16,48} \mathrm{Ac}-$ cordingly, Penning ionization of $\mathrm{He}^{*}$ or $\mathrm{He}_{2}^{*}$ excitations may be enhanced by the simultaneous migration of the two excitations towards the droplet surface due to repulsive $\mathrm{He}-\mathrm{He}^{*}$ interaction $^{3,15,16}$ while polarization forces steer them towards each other as in the case of charge migration. ${ }^{49}$

The vertical dashed lines at $15 \leq E_{e} \leq 18.6 \mathrm{eV}$ indicate the energies of electrons created by symmetric Penning reactions of $\mathrm{He}^{*}$ in various electronic levels as denoted in the legend. Other asymmetric combinations of excited states such as $\mathrm{He}^{*}\left(1 \mathrm{~s} 2 \mathrm{p}^{1} \mathrm{P}\right)+\mathrm{He}{ }^{*}\left(1 \mathrm{~s} 2 \mathrm{~s}^{1} \mathrm{~S}\right)$ are also possible but omitted in Fig. 7 for the sake of clarity. Penning ionization of the directly excited $1 \mathrm{~s} 2 \mathrm{p}^{1} \mathrm{P}$ droplet state yields an electron energy $E_{e}$ $=2 \times h v-E_{i, \mathrm{He}}$ (blue dashed line) whereas Penning ionization following the relaxation of $\mathrm{He}^{*}$ into atomic levels $1 \mathrm{~s} 2 \mathrm{p}^{3} \mathrm{P}$, $1 \mathrm{~s} 2 \mathrm{~s}^{1} \mathrm{~S}, 1 \mathrm{~s} 2 \mathrm{~s}^{3} \mathrm{~S}$ diminishes the Penning electron energy (light dashed lines).

At $\mathrm{He}$ expansion conditions where small droplets $(N=800)$ are formed (Figs. 7(b) and 7(d)), the PES are dominated by direct ionization or by Penning ionization of the unrelaxed He droplet state, which we cannot distinguish. For larger He droplets $N \gtrsim 4000$ (Figs. 7(a) and 7(c)), Penning ionization of $\mathrm{He}^{*}$ that have relaxed into $1 \mathrm{~s} 2 \mathrm{~s}^{1} \mathrm{~S}$ and lowerlying levels prior to ionization becomes more pronounced. This can be rationalized by the longer migration distances covered by the two $\mathrm{He}^{*}$ excitations in large droplets in order to come close and react. The fact that the PES recorded in coincidence with $\mathrm{He}_{2}^{+}$significantly differ from the ones of $\mathrm{He}^{+}$points to a process where first $\mathrm{He}_{2}^{*}$ excited dimers form and then the Penning reaction occurs. The conceivable alternative process, $\mathrm{He}_{2}^{+}$dimer ion formation after Penning ionization of $\mathrm{He}^{*},{ }^{2}$ would result in an identical PES. The small shift of the Penning peak to lower energies in Fig. 7(c) indicates that Penning ionization involves $\mathrm{He}_{2}^{*}$ in various vibronic levels that reach down to even lower energies than the atomic triplet states (vertical dashed line at $E_{e} \approx 11 \mathrm{eV}$ ). Given the low statistics of our data and the limited energy resolution of the spectra we cannot infer more details about this ionization mechanism.

\section{Inelastic photoelectron-helium collisions}

Finally, we present PES measured at high photon energies $h v>46 \mathrm{eV}$. To the best of our knowledge, no experiments with He nanodroplets at such elevated photon energies have been reported to date. Figure 8(a) depicts typical PES correlating to $\mathrm{He}^{+}$and $\mathrm{He}_{2}^{+}$recorded at $h v=50 \mathrm{eV}$. The corresponding raw image for $\mathrm{He}_{2}^{+}$is shown as an inset. While the photoelectron distribution correlating to $\mathrm{He}_{2}^{+}$is significantly more isotropic than that of $\mathrm{He}^{+}$(Fig. 6), the PES are nearly identical at these high photon energies. In addition to the highest peak from directly emitted electrons with energy (a)
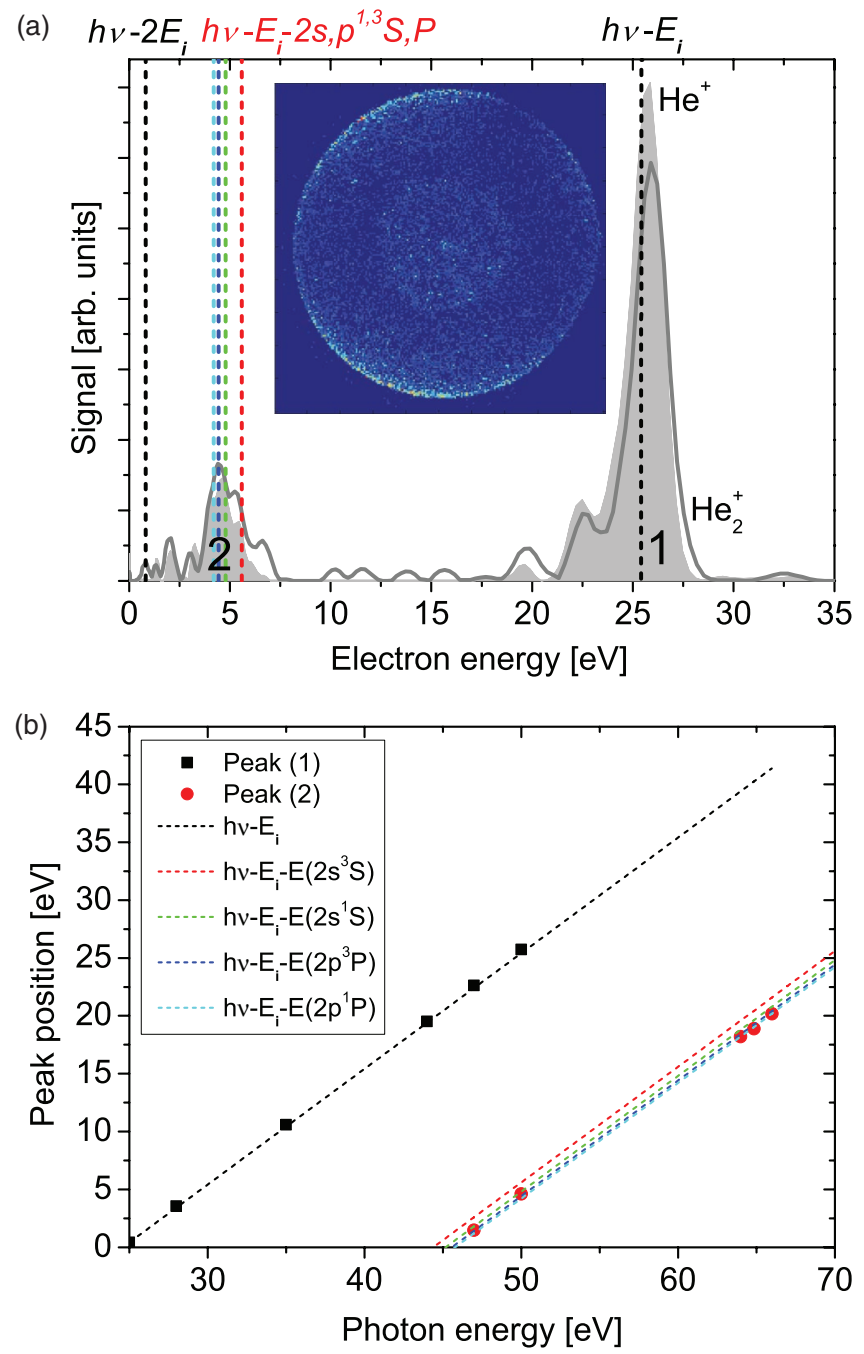

FIG. 8. (a) Photoelectron spectra recorded in coincidence with $\mathrm{He}^{+}$(shaded area) and $\mathrm{He}_{2}^{+}$(solid line) at $h v=50 \mathrm{eV}\left(p_{0}=50\right.$ bars, $\left.T_{0}=23 \mathrm{~K}\right)$. Peak 1 corresponds to electrons created directly by ionization of $\mathrm{He}$ atoms or $\mathrm{He}_{2}$ dimers in the droplets. Peak 2 stems from electrons that lost energy by inelastic collisions with surrounding He atoms. The inset depicts the photoelectron image correlating to $\mathrm{He}_{2}^{+}$. (b) Dependence of the peak positions on $h v$. The dashed lines depict the energies of photoelectrons emitted directly (black) or after energy-loss by inelastic collisions (colored) when considering the He atomic ionization energy $E_{i, \mathrm{He}}$ and the level energies of the $1 \mathrm{~s} 2 \mathrm{~s}^{1,3} \mathrm{~S}$ and $1 \mathrm{~s} 2 \mathrm{p}^{1,3} \mathrm{P}$ atomic levels.

$h v-E_{i, \mathrm{He}}=25.41 \mathrm{eV}$ a second peak appears at energy $E_{E-l o s s}=h v-E_{i, \mathrm{He}}-E_{1 s 2 s, p} \approx 5 \mathrm{eV}$. Here, $E_{1 s 2 s, p}$ stands for the energies of all levels of the 1s2s and $1 \mathrm{~s} 2 \mathrm{p}$ configurations of He atoms $\left({ }^{3} \mathrm{~S},{ }^{1} \mathrm{~S},{ }^{3} \mathrm{P},{ }^{1} \mathrm{P}\right)$ which can be excited by electron impact but remain unresolved in the PES. This lowenergy feature is present at the reduced energy $E_{E-l o s s}$ in all measured spectra where $h v>46 \mathrm{eV}$, as shown in Fig. 8(b). It is due to the loss of kinetic energy of the photoelectrons by inelastic collisions with surrounding $\mathrm{He}$ atoms as they pass through the droplets in a process of the type

$$
e^{-}\left(E_{k i n}\right)+\mathrm{He}\left(1 \mathrm{~s}^{2}\right) \rightarrow \mathrm{He}^{*}+e^{-}\left(E_{k i n}-E_{E-l o s s}\right) .
$$

This interpretation is supported by a vanishing anisotropy parameter $\beta=0.1(3)$ of the PAD in the full range of electron energies covered by peak (2) as opposed to $\beta=1.9(1)$ for peak (1). As expected, the information about the direction of 
emission of the photoelectrons is completely lost by the inelastic electron-He collisions. Our interpretation is in line with previous studies on a solid $\mathrm{Ne}$ surface and on small $(N \sim$ 300) Ne clusters. ${ }^{28,50}$ The appearance of additional features in the PES of the Ne surface and of Ne clusters as compared to the PES of free $\mathrm{Ne}$ atoms was ascribed to satellite lines resulting from energy loss of the outgoing photoelectrons due to the creation of the lowest cluster excitations ("excitons"). ${ }^{28}$

The probability $P_{\text {inel }}$ for such collisions in our experiments can be estimated using the well-known inelastic scattering cross sections $\sigma_{\text {inel }}{ }^{51} P_{\text {inel }}=\sigma_{\text {inel }} \rho_{\mathrm{He}_{N}} R_{\mathrm{He}_{N}}$. Here, $\rho_{\mathrm{He}_{N}}=0.0218 \AA^{-3}$ denotes the density of He droplets $^{52}$ and $R_{\mathrm{He}_{N}}=\left(3 N /\left(4 \pi \rho_{\mathrm{He}_{N}}\right)\right)^{1 / 3}=32 \AA$ is the droplet radius for a mean droplet size $N=2900$. For $h v=50 \mathrm{eV}$ we obtain $P_{\text {inel }} \approx 6 \%$ when summing over all the relevant channels, which roughly matches the ratio of areas of peaks (2) and (1) amounting to about $20 \%$. When estimating the probability of ionizing $\mathrm{He}$ by collisions with photoemitted electrons in the same way, we find $P_{i o n} \approx 1 \%-2 \%$. Unfortunately, this falls below the noise level in our measurements. Besides, in ionizing electron collisions the excess energy is arbitrarily divided between the primary and secondary electrons which leads to a featureless background in the PES instead of a sharp peak as in the case of electron impact excitation.

Note that the elastic electron-He scattering cross section is even larger than the total inelastic cross section for energyloss collisions by a factor $30 .{ }^{53}$ This further confirms our interpretation that the $\mathrm{He}^{+}$atomic ions, for which we measure a very pronounced anisotropy of the coincident electron distribution, stem from the atomic He component accompanying the He droplet beam.

\section{CONCLUSION}

Using velocity-map imaging photoelectron-photoion coincidence (VMI-PEPICO) measurements we have investigated the photoionization dynamics of pure He nanodroplets in the regimes of direct ionization and autoionization. We present photoelectron distributions measured in coincidence with the most abundant ion masses $\mathrm{He}^{+}, \mathrm{He}_{2}^{+}$, and $\mathrm{He}_{3}^{+}$in a wide range of photon energies. The $\mathrm{He}_{2}^{+}$mass-correlated photoelectron spectra are interpreted in terms of contributions from ionized $\mathrm{He}$ droplets that relax to form $\mathrm{He}_{2}^{+}$and from vertically ionized pairs of nearest neighboring $\mathrm{He}$ atoms. The highly anisotropic PAD of the electrons recorded in coincidence with $\mathrm{He}^{+}$indicate that free $\mathrm{He}$ atoms accompanying the droplet beam contribute overwhelmingly to the $\mathrm{He}^{+}$signal. In contrast, PAD of electrons detected in coincidence with $\mathrm{He}_{2}^{+}$ and $\mathrm{He}_{3}^{+}$display significantly reduced anisotropy, presumably due to scattering of the outgoing photoelectron from the $\mathrm{He}$ droplet.

In the regime of pure droplet excitation we measure ionization signals which indicate multiple excitation of the droplets that decay by Penning-like ionization even in the range of small droplets $(N \lesssim 20000)$. Future studies at higher photon fluxes as available at free electron lasers will give more insight into the dynamics of multiple and collective excitations. At high photon energies, we observe electron energy-loss processes by inelastic collisions of the photoelec- trons with $\mathrm{He}$ atoms in the droplets. Such multiple scattering of photoelectrons in clusters is expected to have a severe effect on photoelectron distributions measured in free electron laser experiments.

\section{ACKNOWLEDGMENTS}

The authors gratefully acknowledge support by the staff of Elettra-Sincrotrone Trieste for providing high quality light and P. Piseri and C. Grazioli for technical assistance with the VMI-PEPICO detector. Furthermore, we thank the Deutsche Forschungsgemeinschaft and the Swiss National Science Foundation (Grant No. 200020_140396) for financial support.

${ }^{1}$ A. Stace and D. Bernard, Chem. Phys. Lett. 146, 531 (1988).

${ }^{2}$ H. Buchenau, J. P. Toennies, and J. A. Northby, J. Chem. Phys. 95, 8134 (1991).

${ }^{3}$ A. Scheidemann, B. Schilling, and J. P. Toennies, J. Chem. Phys. 97, 2128 (1993).

${ }^{4}$ N. Halberstadt and K. C. Janda, Chem. Phys. Lett. 282, 409 (1998).

${ }^{5}$ J. Seong, K. C. Janda, N. Halberstadt, and F. Spiegelmann, J. Chem. Phys. 109, 10873 (1998).

${ }^{6}$ R. Fröchtenicht, U. Henne, J. P. Toennies, A. Ding, M. Fieber-Erdmann, and T. Drewello, J. Chem. Phys. 104, 2548 (1996).

${ }^{7}$ D. S. Peterka, A. Lindinger, L. Poisson, M. Ahmed, and D. M. Neumark, Phys. Rev. Lett. 91, 043401 (2003).

${ }^{8}$ D. S. Peterka, J. H. Kim, C. C. Wang, L. Poisson, and D. M. Neumark, J. Phys. Chem. A 111, 7449 (2007).

${ }^{9}$ K. von Haeften, A. R. B. de Castro, M. Joppien, L. Moussavizadeh, R. von Pietrowski, and T. Möller, Phys. Rev. Lett. 78, 4371 (1997).

${ }^{10}$ K. von Haeften, T. Laarmann, H. Wabnitz, and T. Möller, J. Phys. B 38, S373 (2005).

${ }^{11}$ K. von Haeften, T. Laarmann, H. Wabnitz, T. Möller, and K. Fink, J. Phys. Chem. A 115, 7316 (2011).

${ }^{12}$ O. Kornilov, C. C. Wang, O. Bünermann, A. T. Healy, M. Leonard, C. Peng, S. R. Leone, D. M. Neumark, and O. Gessner, J. Phys. Chem. A 114, 1437 (2010)

${ }^{13}$ O. Kornilov, O. Bünermann, D. J. Haxton, S. R. Leone, D. M. Neumark, and O. Gessner, J. Phys. Chem. A 115, 7891 (2011).

${ }^{14}$ O. Bünermann, O. Kornilov, S. R. Leone, D. M. Neumark, and O. Gessner, IEEE J. Sel. Top. Quantum Electron. 18, 308 (2012).

${ }^{15}$ O. Bünermann, O. Kornilov, D. J. Haxton, S. R. Leone, D. M. Neumark, and O. Gessner, J. Chem. Phys. 137, 214302 (2012).

${ }^{16}$ D. Buchta, S. R. Krishnan, N. B. Brauer, M. Drabbels, P. O’Keeffe, M. Devetta, M. Di Fraia, C. Callegari, R. Richter, M. Coreno, K. C. Prince, F. Stienkemeier, R. Moshammer, and M. Mudrich, J. Phys. Chem. A 117, 4394 (2013).

${ }^{17}$ J. H. Kim, D. S. Peterka, C. C. Wang, and D. M. Neumark, J. Chem. Phys. 124, 214301 (2006).

${ }^{18}$ M. Joppien, R. Karnbach, and T. Möller, Phys. Rev. Lett. 71, 2654 (1993).

${ }^{19}$ J. A. Northby and T. M. Sanders, Phys. Rev. Lett. 18, 1184 (1967).

${ }^{20}$ M. Rosenblit and J. Jortner, Phys. Rev. Lett. 75, 4079 (1995).

${ }^{21}$ K. von Haeften, T. Laarmann, H. Wabnitz, and T. Möller, Phys. Rev. Lett. 88, 233401 (2002).

${ }^{22}$ M. Fárník and J. P. Toennies, J. Chem. Phys. 118, 4176 (2003).

${ }^{23}$ H. Zhang, D. Rolles, Z. D. Pešić, J. D. Bozek, and N. Berrah, Phys. Rev. A 78, 063201 (2008).

${ }^{24}$ T. Pflüger, A. Senftleben, X. Ren, A. Dorn, and J. Ullrich, Phys. Rev. Lett. 107, 223201 (2011).

${ }^{25}$ G. Öhrwall, M. Tchaplyguine, M. Gisselbrecht, M. Lundwall, R. Feifel, T. Rander, J. Schulz, R. R. T. Marinho, A. Lindgren, S. L. Sorensen, S. Svensson, and O. Björneholm, J. Phys. B 36, 3937 (2003).

${ }^{26}$ D. Rolles, H. Zhang, Z. D. Pešić, R. C. Bilodeau, A. Wills, E. Kukk, B. S. Rude, G. D. Ackerman, J. D. Bozek, R. Díez Muiño, F. J. García de Abajo, and N. Berrah, Phys. Rev. A 75, 031201(R) (2007).

${ }^{27}$ K. von Haeften, T. Laarmann, H. Wabnitz, and T. Möller, Phys. Rev. Lett. 87, 153403 (2001).

${ }^{28}$ U. Hergenhahn, A. Kolmakov, M. Riedler, A. de Castro, O. Löfken, and T. Möller, Chem. Phys. Lett. 351, 235 (2002). 
${ }^{29}$ P. O'Keeffe, P. Bolognesi, M. Coreno, A. Moise, R. Richter, G. Cautero, L. Stebel, R. Sergo, L. Pravica, Y. Ovcharenko, and L. Avaldi, Rev. Sci. Instrum. 82, 033109 (2011).

${ }^{30}$ J. P. Toennies and A. F. Vilesov, Angew. Chem., Int. Ed. 43, 2622 (2004).

${ }^{31}$ F. Stienkemeier and K. Lehmann, J. Phys. B 39, R127 (2006).

${ }^{32}$ M. J. J. Vrakking, Rev. Sci. Instrum. 72, 4084 (2001).

${ }^{33}$ G. A. Garcia, L. Nahon, and I. Powis, Rev. Sci. Instrum. 75, 4989 (2004).

${ }^{34}$ D. S. Peterka, J. H. Kim, C. C. Wang, and D. M. Neumark, J. Phys. Chem. B 110, 19945 (2006).

${ }^{35}$ J.-E. Rubensson, A. Moise, A. Mihelič, K. Bučar, M. Žitnik, and R. Richter, Phys. Rev. A 81, 062510 (2010).

${ }^{36}$ C. C. Wang, O. Kornilov, O. Gessner, J. H. Kim, D. S. Peterka, and D. M. Neumark, J. Phys. Chem. A 112, 9356 (2008).

${ }^{37}$ L. Fechner, B. Grüner, A. Sieg, C. Callegari, F. Ancilotto, F. Stienkemeier, and M. Mudrich, Phys. Chem. Chem. Phys. 14, 3843 (2012).

${ }^{38}$ A. R. Janzen and R. A. Aziz, J. Chem. Phys. 107, 914 (1997).

${ }^{39}$ A. Carrington, C. H. Pyne, and P. J. Knowles, J. Chem. Phys. 102, 5979 (1995).

${ }^{40}$ R. N. Zare, Mol. Photochem. 4, 1 (1972).

${ }^{41}$ T. Havermeier, T. Jahnke, K. Kreidi, R. Wallauer, S. Voss, M. Schöffler, S. Schössler, L. Foucar, N. Neumann, J. Titze, H. Sann, M. Kühnel, J. Voigtsberger, A. Malakzadeh, N. Sisourat, W. Schöllkopf, H. SchmidtBöcking, R. E. Grisenti, and R. Dörner, Phys. Rev. Lett. 104, 153401 (2010).
${ }^{42}$ A. I. Kuleff, K. Gokhberg, S. Kopelke, and L. S. Cederbaum, Phys. Rev. Lett. 105, 043004 (2010).

${ }^{43}$ C. Müller, M. A. Macovei, and A. B. Voitkiv, Phys. Rev. A 84, 055401 (2011).

${ }^{44}$ J. W. Keto, F. J. Soley, M. Stockton, and W. A. Fitzsimmons, Phys. Rev. A 10, 872 (1974).

${ }^{45}$ S. G. Kafanov, A. Y. Parshina, and I. A. Todoshchenko, JETP 91, 991 (2000).

${ }^{46}$ D. Zmeev, F. Papkour, P. Walmsley, A. Golov, P. McClintock, S. Fisher, W. Guo, D. McKinsey, G. Ihas, and W. Vinen, J. Low Temp. Phys. 171, 207 (2013).

${ }^{47}$ M. Müller, A. Merz, M.-W. Ruf, H. Hotop, W. Meyer, and M. Movre, Z. Phys. D: At. Mol. Clust. 21, 89 (1991).

${ }^{48}$ A. A. Scheidemann, V. V. Kresin, and H. Hess, J. Chem. Phys. 107, 2839 (1997).

${ }^{49}$ W. Lewis, C. Lindsay, R. Bemish, and R. Miller, J. Am. Chem. Soc. 127, 7235 (2005).

${ }^{50}$ H. H. Farrell and M. Strongin, Phys. Rev. B 6, 4711 (1972).

${ }^{51}$ Y. Ralchenko, R. Janev, T. Kato, D. Fursa, I. Bray, and F. de Heer, At. Data Nucl. Data Tables 94, 603 (2008).

${ }^{52}$ J. Harms, J. P. Toennies, and F. Dalfovo, Phys. Rev. B 58, 3341 (1998).

${ }^{53}$ D. F. Register, S. Trajmar, and S. K. Srivastava, Phys. Rev. A 21, 1134 (1980). 